\title{
MEMORIA OPERACIONAL VERBAL Y DIFERENCIAS DE READING SPAN EN ESCOLARES
}

\section{OPERATIONAL VERBAL MEMORY AND DIFFERENTIATE READING'S SPAN IN STUDENTS}

\author{
Nicolas Medina C. ${ }^{1}$, Rita Guillen R., Edson Françozo ${ }^{2}$ \\ InStituto de Estudos da Linguagem \\ Universidade Estadual de Campinas, São Paulo, Brasil \\ (RECIBIDO EL 02/02/2009, ACEPTADo EL 24/06/2009)
}

\begin{abstract}
RESUMEN
El propósito de este estudio fue analizar la memoria operacional (working memory) como un modelo cognitivo implicado en la tarea lexical y en la diferenciación individual de las habilidades en la lectura. Los sujetos fueron 163 escolares de enseñanza fundamental, que asisten a escuelas públicas y particulares de la ciudad de Campinas, San Pablo, Brasil. En primer lugar, elaboramos una nueva versión del test reading span de acuerdo con el vocabulario del portugués brasilero, para medir las diferencias individuales de la capacidad de la memoria operacional verbal, según los criterios sugeridos por Daneman y Carpenter (1980), adaptado para escolares de enseñanza fundamental, cuya validez instrumental fue verificada correlacionándolo con el subtest de número del WISC, en la modalidad directa e inversa. Los resultados constataron una correlación positiva significativa $(\mathrm{rRHO}=0,47)$ entre la nueva versión del test reading span que elaboramos y el subtest de número del WISC, en la modalidad invertida, lo que demostraría que ambos tests contienen el componente de procesamiento de la memoria operacional. Consecuentemente, el nuevo test que proponemos estaría habilitado para establecer diferencias individuales de las capacidades de lectura en sujetos con las mismas características del grupo estudiado.
\end{abstract}

Palabras clave: Memoria operacional, tarea de capacidad de lectura, comprensión de lectura, diferencias individuales en la lectura, recursos compartidos, procesamiento y almacenamiento.

\footnotetext{
ABSTRACT

The purpose of this work was to analyze the working memory like a cognitive model envolved in lexical tasks and individual differentiation on capacity of Reading. The subjects were 163 students of public and particular schools. In this study we elaborated a new "reading

1 Docente investigador del Instituto de Investigaciones Psicológicas de la Facultad de Psicología, Universidad Nacional Mayor de San Marcos, Lima-Perú. E-mail: medina0712@yahoo.com

2 Docente, Professor Asociado Instituto de Estudos da Linguagem. E-mail: edson@iel.unicamp.br
} 
span test" agreement with suggestions from Daneman and Carpenter (1980), adapted to children students. That instrument was correlated with the subtest of Number from WISC, inverted form. The validity assessment between both tests was made with Spearman's coefficient of correlation. The consequence of that assessment showed rRHO $=0,47$; it should prove both tests contain the function of processing (or computation) proper of the working memorysystem. Then, that "reading span test" is an adequate instrument to measure individual differences about capacity of reading only in similar subjects like those we studied.

Keywords: working memory; reading span task; reading comprehension; individual differences; resource sharing; processing and storage.

\section{INTRODUCCIÓN}

Desde el punto de vista de la psicolingüística, se supone que en el proceso de comprensión de oraciones participan determinados mecanismos cognitivos que lo explicarían, al menos parcialmente. En la actualidad existen muchas evidencias empíricas que constatan que el constructo de la memoria operacional (working memory) es una hipótesis que explicaría adecuadamente la intervención de específicos mecanismos cognitivos en la comprensión de oraciones; es decir, por medio de ese constructo las unidades de información lingüística serían tratadas computacionalmente por la doble función simultánea con que cuenta la memoria operacional: el almacenamiento y el procesamiento. De modo que, frente a una tarea cognitiva compleja en el "momento real" (online), como la lectura, esas funciones son activadas.

Tradicionalmente, la memoria a corto plazo fue concebida como un almacén temporal que conservaba una cantidad limitada de ítems, utilizando estrategias simples de repetición (rehearsal) o agrupamiento de ítems (chunking). Mientras que la memoria operacional es considerada como un espacio computacional más flexible, con una cantidad limitada de recursos atencionales requeridos para el procesamiento y manipulación de la información, además del almacenamiento de los productos parciales y finales de esos procesos.

\section{Rasgos característicos de la memoria operacional}

Ruchkin et al. (2003) caracterizaron la memoria operacional de conciso, señalando que es un sistema cognitivo que mantiene información de una tarea importante en un estado activo durante el desempeño de esa tarea. Es decir, la memoria operacional funciona como un espacio de trabajo en el cual la información sensorial recientemente adquirida y la información de la memoria a largo plazo son procesadas para facilitar la acción (e. g., almacenamiento, computación, toma de decisión). El constructo de la memoria operacional se desarrolló en base al modelo de la memoria a corto plazo, que formó parte del modelo modal propuesto por Atkinson y Shiffrin $(1968,1983)$, quienes postularon que el sistema de la memoria a corto plazo recibe input del almacenamiento sensorial, y transfiere y recupera información del almacén al largo plazo. No obstante que el modelo modal explique varios resultados empíricos relacionados con el funcionamiento de la memoria, no provee una correcta explicación sobre cómo interactúan las memorias de corto plazo y de largo plazo, ni predice correctamente el desempeño en determinados experimentos con doble tarea, ni da una explicación adecuada de los desempeños de la memoria de sujetos amnésicos. 
Baddeley y Hitch $(1974,1983)$ resolvieron muchos de esos problemas proponiendo el modelo de la memoria operacional, considerándolo como un sistema multicomponente, en la medida que comprende varios subsistemas: un ejecutivo central, que controla el procesamiento consciente de la información, además de un par de subsistemas subsidiarios como el lazo fonológico (o articulatorio) y la agenda visoespacial, que almacenan temporalmente información fonológica e información visoespacial, respectivamente. En una revisión reciente de este modelo, Baddeley (2000) adicionó un cuarto subsistema de almacenamiento de corto plazo: el almacén episódico (episodic buffer) que forma una interface entre el almacenamiento fonológico a corto plazo, el almacén visoespacial a corto plazo y la memoria a largo plazo. El almacenamiento episódico aumenta la capacidad de la memoria operacional, manteniendo el material integrado de acuerdo con las situaciones y eventos concretos. El ejecutivo central es considerado como un mecanismo de control de la atención, sin capacidad de almacenamiento. Un aspecto importante del modelo de Baddeley es que los varios subsistemas generan diferentes recursos de procesamiento que pueden, en alguna medida, funcionar independientemente.

\section{Modelos de memoria operacional}

Basados en el trabajo original de Baddeley y Hitch $(1974,1983)$, surgieron otros modelos de memoria operacional que explicaron de modo diferente el funcionamiento de los mecanismos de procesamiento y almacenamiento, frente a una tarea de comprensión de lectura (ver Conway y Engle, 1996; Engle y Oransky, 1999; Fedorenko, Gibson y Rhode, 2006; Friedman y Miyake, 2004; Miyake y Shah, 1999). Al respecto cabe señalar que el primer modelo fue justamente el que propusieron Baddeley y Hitch $(1974,1983)$ quienes destacan el carácter multicomponente de la memoria operacional, considerando que los mecanismos de procesamiento y de almacenamiento son independientes, en la medida que el procesamiento es controlado por el ejecutivo central y el almacenamiento es una función del lazo fonológico o articulatorio.

El segundo modelo se relaciona con la explicación proporcionada por Daneman y Carpenter (1980), y desarrollada por Just y Carpenter (1992), conocido como el "modelo de doble función en un solo sistema", mediante el cual se explica que las funciones del procesamiento y almacenamiento cuentan con un espacio común de recursos, que es compartido por esas dos funciones. De esa manera, se supone que el recurso total disponible para el almacenamiento depende de la eficacia del procesamiento. En relación a ello, Daneman y Carpenter (1980) afirmaron que los lectores con procesos más eficientes en la lectura de oraciones tendrían mayor capacidad de memoria operacional disponible para almacenar más ítems de información que los lectores con procesamiento menos eficientes.

Un tercer modelo postula que la capacidad de la memoria operacional se basa en la atención controlada, asumiendo que la capacidad de la memoria operacional no se refiere al almacenamiento o memoria per se, sino que constituye una capacidad para la atención sustentada y controlada frente a la interferencia o distracción. Ese modelo es conocido como la hipótesis de la capacidad general (Conway y Engle, 1996), que afirma que los sujetos con alta capacidad de memoria operacional tienen más recursos atencionales que los sujetos con baja capacidad. En ese sentido, las diferencias individuales determinadas por 
las medidas de la capacidad de la memoria operacional, reflejan básicamente diferencias en la capacidad para el procesamiento controlado, que se activan por situaciones que demandan la intervención de la atención controlada. De acuerdo con Engle y Oransky (1999), la atención controlada se hace necesaria en las siguientes circunstancias: cuando las metas de las tareas podrían perderse a menos que sean mantenidas activamente en la memoria operacional; cuando las acciones en competencia deben ser planificadas; cuando los conflictos de acciones deben ser resueltos; cuando es importante mantener alguna información frente a la distracción o interferencia, es importante suprimir información sin importancia para realizar una tarea (Engle y Oransky, 1999: 539).

Caplan y Waters (1999) mencionaron un modelo de memoria operacional con un componente más de los que originalmente poseían, que actuaría como un recurso de procesamiento especificando la estructura sintáctica de una oración, para determinar el significado de esa oración. Inclusive esos autores hablaron de la posibilidad de que el "sistema de memoria operacional verbal" estaría abierto para adicionar otros subsistemas dedicados a diferentes tipos de tareas verbales (Caplan y Waters, 1999: 78).

Los modelos arriba mencionados concuerdan en la idea central que la memoria operacional es un sistema temporal, con capacidad limitada, esencialmente activo, que procesa información lingüística para comprenderla, y es requerida por una tarea compleja online, como en el caso de la lectura.

\section{Sobre la medición de la memoria operacional}

De acuerdo con uno de los propósitos de este estudio, que es elaborar una versión nueva del test de reading span según los criterios sugeridos por Daneman y Carpenter (1980), cabe señalar los aspectos relevantes de la concepción de estas autoras sobre memoria operacional. Así, Daneman y Carpenter (1980) postularon que la fuente cognitiva implicada en la comprensión de lectura está representada por el intercambio funcional entre el procesamiento y el almacenamiento, que compiten en un espacio de trabajo limitado de la memoria operacional. De modo que se puede decir que el lector eficiente tendría procesos más eficientes, que le permitirían poseer más capacidad para la retención de información. De igual modo, el lector eficiente podría requerir menor procesamiento que un lector deficiente. Eso implicaría que en los lectores eficientes serían eliminados pasos intermedios de las fases de procesamiento, tales como la codificación, acceso lexical, análisis gramatical, inferencia e integración. Esa eficiencia significaría que el buen lector tendría pocas demandas computacionales en la memoria operacional; por eso, el lector eficiente tendría mayor capacidad para almacenar los productos intermedios y finales necesarios del procesamiento de la lectura. Del mismo modo, los procesos más eficientes también serían funcionalmente más rápidos y, de facto, los mejores lectores son más rápidos en tareas relacionadas con la lectura.

Daneman y Carpenter también indican que existen varios estudios sobre pruebas padronizadas que miden la capacidad de memoria de dígitos (digit span), que no establecen diferencias sistemáticas entre eficientes y deficientes lectores, clasificados mediante una prueba general de comprensión de lectura. La explicación de esa falta de correlación podría ser la siguiente: que las pruebas de la capacidad de memoria de dígitos no evalúan 
satisfactoriamente el componente de procesamiento de la memoria operacional, ya que esas pruebas implicarían procesos simples de repetición (rehearsal) de los ítems de información. Se supone que una tarea que demanda procesamiento más complejo necesitará mayor intercambio funcional entre procesamiento y almacenamiento.

Basadas en esos supuestos, Daneman y Carpenter (1980) elaboraron un instrumento que evaluó ambas funciones de la memoria operacional: el procesamiento y el almacenamiento, al cual denominaron Test de Reading Span (o test de la capacidad de lectura). El formato de ese test fue similar a las pruebas tradicionales de digit span (capacidad de la memoria de dígitos). En relación a la fundamentación teórica del test de reading span, Just y Carpenter (1992) señalaron que la función de la memoria operacional, además de almacenar información, abarca operaciones de computación, explicando al mismo tiempo que las computaciones son manipulaciones simbólicas que se expresan a través de operaciones de comparación, recuperación y operaciones lógicas y numéricas, que constituyen el núcleo de la cognición humana. En ese sentido, esos procesos (o computaciones) cumplen un papel importante en la comprensión del lenguaje. Por ello se puede decir que esos procesos, junto con los recursos de almacenamiento, establecen la naturaleza de la memoria operacional verbal.

Cabe recordar que Baddeley y Hitch $(1974,1983)$ indicaron que la memoria operacional es el locus de la computación, y demostraron que la habilidad para comprender oraciones disminuyó rápidamente cuando los lectores tuvieron que codificar (durante la lectura) simultáneamente varios dígitos para evocarlos después, lo cual implicó un intercambio funcional entre el almacenamiento y el procesamiento, y ese hecho sugirió que esas dos funciones comparten un espacio común de recursos. Consecuentemente, Just y Carpenter (1992) afirmaron que hay razones conceptuales y empíricas que sustentan la noción de la doble función de la memoria operacional (procesamiento y almacenamiento) dentro de un solo sistema. Esa noción mantuvo una diferencia en relación a la idea de memoria operacional propuesta por Baddeley (1986). De modo que, desde el punto de vista de Just y Carpenter (1992), la memoria operacional verbal se refiere a un grupo de procesos y recursos que participan en la comprensión del lenguaje. Mientras que en la teoría original de Baddeley, la memoria operacional pone énfasis en su aspecto estructural, considerando dos componentes relevantes: el lazo articulatorio o fonológico y el ejecutivo central. En el modelo de Just y Carpenter la memoria operacional está estrechamente relacionada con el funcionamiento del ejecutivo central, que trata del proceso de comprensión del lenguaje, y no incluye buffers de modalidad específica, como el lazo articulatorio.

\section{Memoria operacional y diferencias individuales}

En el presente estudio estamos de acuerdo con la tesis que afirma que la naturaleza de la habilidad que tiene una persona en la comprensión del lenguaje depende de su capacidad de la memoria operacional. Siendo así, se supone que cuando la demanda de una tarea compleja es grande y se aproxima al límite de la capacidad de la memoria operacional, entonces el individuo o tendrá dificultad para procesar adecuadamente información o para almacenar productos parciales del proceso. De modo que las diferencias individuales en la capacidad de la memoria operacional, frecuentemente, son evaluadas con tareas 
simultáneas que demandan mecanismos de procesamiento y almacenamiento. Al respecto, varios autores confirmaron que la memoria operacional cumple un papel importante en el desempeño de la lectura (Baddeley, Logie, Nimmo-Smith y Brereton, 1985; Cain, Oakhill y Bryant, 2004; Daneman y Carpenter, 1980, 1982, 1983; Daneman y Merikle, 1996; Dixon, LeFevre y Twilley, 1988; King y Just, 1991; Lima y Françozo, 2001; Turner y Engle, 1989). Se supone que eso se debe a la dinámica interna de la memoria operacional, en la que las funciones de procesamiento y almacenamiento compiten por una capacidad limitada, que debe ser compartida por ambas funciones. Así, cuanto mayor es la demanda de procesamiento, se consume más espacio disponible, disminuyendo la cantidad de información que podría ser almacenada y retenida en la memoria operacional. Esa forma de compartir diferencialmente la capacidad limitada de la memoria operacional, entre el procesamiento y el almacenamiento, se realiza de manera distinta en cada individuo, de modo que las diferencias individuales en la memoria operacional expresan diferencias en la eficiencia de la comprensión de lectura.

Basadas en esos presupuestos, Daneman y Carpenter (1980) elaboraron el Test de Reading Span, por medio del cual fue posible evaluar la capacidad de la memoria operacional, mientras que el sujeto ejecuta una tarea de lectura. La aplicación del Test de Reading Span sugiere que el sujeto lea un grupo de oraciones y al finalizar cada grupo debe evocar las palabras finales de cada oración. De esa manera, la capacidad de la memoria operacional es definida como el mayor grupo de palabras finales recordadas correctamente.

Cabe señalar que la doble tarea que demanda el Test de Reading Span (leer y retener), exige la activación de ambas funciones de la memoria operacional: el procesamiento y el almacenamiento. Desde el punto de vista ecológico, esa actividad constituye una aproximación más real a las complejas tareas cognitivas de la actividad cotidiana. En ese sentido, el Test de Reading Span es un buen predictor de la tarea de comprensión de lectura que los tests tradicionales, que solo miden la capacidad de la memoria a corto plazo, que demanda simplemente almacenamiento de la información.

\section{Estudio correlacional de la memoria operacional}

La elaboración de nuestra nueva versión del Test de Reading Span demandó su validación, es decir, si ese nuevo instrumento mediría lo que pretende medir. En ese sentido, en este trabajo nos propusimos correlacionar el nuevo Test de Reading Span con el Subtest Memoria de Dígitos de la Escala Wechsler de Inteligencia para niños (Wechsler Intelligence Scale for Children-WISC), que presenta dos modalidades: una que mide la memoria inmediata en forma directa y otra que mide la memoria inmediata en forma inversa. La correlación más importante será entre el nuevo Test de Reading Span que estamos proponiendo en este estudio y los resultados del Subtest de Memoria de Dígitos del WISC en la modalidad inversa. Esa correlación intentará comprobar la implicancia del raciocinio, como una actividad cognitiva presente en el procesamiento, tanto en la memoria operacional (reading span) como en la memoria de dígitos (digit span) en la forma inversa del WISC, por medio del cual se constataría la presencia del función de procesamiento en el nuevo test que intentamos validar. 
En relación a la implicancia de raciocinio en la memoria operacional, Süß et al. (2002) señalaron que la memoria operacional participa en forma significativa en la realización de tareas complejas, aplicando habilidades de raciocinio que constituyen un subconstructo importante dentro de la estructura de la inteligencia. El concepto general de raciocinio fue entendido como un proceso cognitivo relativamente complejo, que consiste en la secuencia organizada de pasos que deben ser planificados adecuadamente con la finalidad de alcanzar una meta (Süß et al., 2002).

Por su parte, Schofield y Ashman (1986) indicaron que los test de digit span adoptan dos formas: la medida de la capacidad de retener dígitos en forma inversa (backward digit span) y la medida de la capacidad de retener dígitos en forma directa (forward digit span), afirmando que la primera forma implica procesamiento más complicado que la segunda forma. Esa distinción se fundamenta en la teoría de los dos niveles de las habilidades mentales de Jensen, que menciona dos niveles mentales: el Nivel I, relacionado con la habilidad de la memoria para repetir una lista de dígitos tal como fueron escuchados (forward digit span), que se caracteriza por la falta de operaciones de elaboración, transformación o manipulación de los ítems ingresados; y el Nivel II, relacionado con la habilidad de la memoria para repetir una lista de dígitos en forma inversa (backward digit span), que demanda la participación de la habilidad de raciocinio y de las operaciones de transformación y manipulación mental de los datos ingresados en el Nivel I.

Tomando en cuenta que el factor $\mathrm{g}$ de la inteligencia refleja las habilidades del Nivel II, Jensen y Figueroa (1975) demostraron que las mediciones de la capacidad de dígitos en la forma inversa (backward digit span) correlacionan significativamente con CI, lo que no se verifica con la medición de la capacidad de dígitos en la forma directa (forward digit span). En ese sentido, se confirmó que la capacidad de retención de dígitos (digit span), en las formas directa e inversa, no miden una entidad unitaria, sino dos procesos diferentes. Siendo así, el digit span en la forma inversa implicaría habilidades de raciocinio relativo a la manipulación mental o transformación de los datos ingresados a la memoria inmediata, mientras que el digit span, en la forma directa, no.

Varios estudios han demostrado que las mediciones simples de la capacidad de la memoria a corto plazo (e.g. retención y evocación de una serie de dígitos) no correlacionan consistentemente con medidas de comprensión de lectura (Daneman y Carpenter, 1980; Engle y Oransky, 1999). Se supone que eso se debe a que en las tareas simples de la memoria de dígitos (digit span) se activa únicamente el componente de almacenamiento de la memoria inmediata. Mientras que en una tarea compleja, como en el Test de Reading Span se activan simultáneamente los componentes de procesamiento y almacenamiento, reflejando así una mejor aproximación a los requerimientos de la memoria operacional, relacionada con tareas de procesamiento del lenguaje. Al respecto, Waters y Caplan (1996) constataron que el procesamiento es el principal componente que permite la correlación entre los test de reading span y las pruebas de compresión de lectura.

Cabe señalar que el Test de Reading Span elaborado por Daneman y Carpenter (1980) presenta alta correlación con los aspectos relativos a la comprensión de lectura del test verbal denominado Test de Aptitud Escolar (Scholastic Aptitude Test - SAT), mostrando índices de correlación entre 0.5 e 0.6 (Daneman y Carpenter, 1980; Just et al., 1996; Masson e Miller, 
1983). Bajo esas evidencias, el Test de Reading Span es un instrumento que puede predecir con éxito el desempeño en la comprensión de lectura y, al mismo tiempo, puede medir diferencias individuales tanto en la eficiencia de la lectura como en la capacidad de almacenamiento de la memoria operacional (Dixon et al., 1988; MacDonald et al., 1992).

\section{Factores socioeconómicos y la capacidad en la lectura}

Cabe destacar que los psicólogos experimentalistas y los antropólogos han constatado que los factores socioeconómicos influyen significativamente en la estructuración de la naturaleza de determinadas funciones psicológicas, principalmente en el de los procesos cognitivos superiores. Por ejemplo, Cole (1999) refirió que la distinción entre procesos elementales y superiores fue reconocida por Wundt, fundador de la psicología experimental, y consideró que era imposible estudiar las funciones psíquicas superiores libre de las experiencias organizadas culturalmente, que son diferentes de un individuo y otro, y de una sociedad y otra. De modo que Wundt sugirió que los científicos de la mente deberían utilizar las evidencias etnológicas y del folclor para descubrir las propiedades adecuadas de la mente, construidas sobre la base de procesos elementales que él estudió en el laboratorio. En esa perspectiva, Cole (1999) concedió importancia a las condiciones sociales y culturales como factores que no deberían ser ignorados en el estudio de las propiedades de los procesos cognitivos superiores, y al mismo tiempo recordó que Boaz afirmó, hace medio siglo, que era impensable la existencia de una mente absolutamente independiente de las condiciones de la vida (Cole, 1999: 663).

Contrario al punto de vista arriba mencionado, es pertinente mencionar un trabajo realizado por Engel, Santos, Martin y Gathercole (2007), donde formularon la hipótesis que afirma que las habilidades de la memoria operacional son independientes de los factores medioambientales, tales como el factor socioeconómico o cultural. Para probar esa hipótesis, estudiaron a un grupo de niños brasileros de 6-7 años, pertenecientes a familias con estatus socioeconómico bajo y alto. La memoria operacional de los niños fue evaluada con instrumentos que midieron su memoria verbal a corto plazo, su capacidad verbal compleja y su vocabulario. Los resultados muestran que los niños del grupo socioeconómico bajo obtuvieron significativamente bajos puntajes en los test de vocabulario, pero no en las medidas de la memoria verbal a corto plazo, comparadas con sus pares de estatus socioeconómico alto. También ambos grupos tuvieron igual desempeño en el test de memoria de dígitos en la forma invertida. Esos resultados indicaron que los test de memoria verbal a corto plazo y de la memoria de dígitos invertida providenciaron mediciones de las habilidades cognitivas que no están influenciadas por la cualidad de la condición socioeconómica del niño. En un segundo estudio del mismo trabajo, el grupo de niños brasileros previamente estudiados fue comparado con otro grupo de niños inmigrantes de habla portuguesa que vivían en Luxemburgo. Esa comparación fue realizada en base a las mismas medidas de las habilidades cognitivas aplicadas anteriormente. Los resultados constataron que la memoria verbal a corto plazo y la memoria de dígitos invertida también estaban libres de las influencias culturales. En consecuencia, se concluyó que determinados aspectos de la memoria operacional, como la memoria verbal a corto plazo y la memoria invertida de dígitos, están libres de las influencias socioeconómicas y culturales. 
Otro trabajo realizado por Santos (2007) estudió niños brasileros de 5-12 años, pertenecientes a poblaciones urbanas y rurales, administrándoseles pruebas de memoria (memoria operacional y memoria episódica), cuyos resultados no fueron diferentes entre ambos grupos de niños, concluyendo que los resultados de las pruebas de memoria estuvieron libres de la influencia cultural.

En suma, el objetivo principal de este trabajo fue analizar la capacidad en la lectura (reading span) y sus niveles, en un grupo de niños escolarizados de enseñanza fundamental, teniendo como base teórica el modelo de la memoria operacional. Con ese propósito elaboramos previamente un nuevo test de reading span Test de Reading Spanter (1980), adaptado para niños, en la medida que no existía en Brasil un instrumento similar con esas características. Además de eso, procedimos a correlacionar ese nuevo test con el subtest de memoria de dígitos (en la forma inversa) del WISC, con la finalidad de establecer su validez correspondiente.

\section{MÉTODO}

\section{Elaboración de un Test de Reading Span}

Sobre la base de la hipótesis de memoria operacional postulada por Daneman y Carpenter (1980), y siguiendo los criterios sugeridos por las mencionadas autoras para construir el Test de Reading Span, decidimos elaborar una nueva versión del mencionado test, adaptado para niños escolarizados de enseñanza fundamental, con la finalidad de contar con un instrumento que pueda evaluar adecuadamente el nivel de reading span, y además que sea factible de predecir el desempeño en la lectura de los niños. Con ese propósito, trazamos un plan de investigación que nos llevó, paso a paso, a construir finalmente el mencionado test de reading span.

En primer lugar, fue necesario realizar una etapa preliminar de actividades con vista a elaborar las frases que formarían parte del mencionado test. Esas actividades de elaboración de las frases fueron las siguientes:

1. Revisión teórica en relación con el tema de Comprensión de Oraciones, publicados en artículos científicos, enfocados desde la perspectiva psicolingüística, en la que destacan dos puntos de vista: el enfoque clásico simbólico representacionalista y el enfoque conexionista no simbólico (llamado también procesamiento distribuido en paralelo-PDP o red neuronal); además de esos enfoques, hay otra tendencia que destaca el modelo de sistema dinámico, que concibe la naturaleza del lenguaje como expresión de acciones corporales. Toda esa información teórica nos proporcionó una visión general sobre la producción y comprensión de oraciones.

2. Construcción preliminar de 73 frases, con las siguientes características: las frases fueron elaboradas considerando entre siete y diez palabras; teniendo en total cada frase de 17 a 20 sílabas; dichas frases terminaron en substantivos con tres sílabas.

Para elaborar esas frases, se consultó diversas fuentes impresas, que tienen una determinada frecuencia en las tareas de lectura del nivel de enseñanza fundamental, 
tales como: textos escolares de gramática de la lengua portuguesa y textos de lectura; obras literarias (e. g., Sítio do Pica Pau Amarelo, de Monteiro Lobato; Ana Levada da Breca, de Maria Lourdes Krieger; Quatro Mitos Brasileiros, de Mônica Stahel; Lili a Rainha das Escolhas, de Elisa Lucinda; Cara ou Coroa? de Fernando Sabino; entre otros); revistas dirigidas para niños y adolescentes, como Recreio; Diccionario Aurélio de la lengua portuguesa.

3) Esas 73 frases preliminares fueron sometidas a un análisis acerca de su pertinencia lexical y gramatical, por diez profesoras de enseñanza fundamental, de escuelas estatales públicas y particulares, considerando los siguientes criterios:

- que las frases estén bien formuladas;

- que las frases contengan términos del vocabulario diario de los alumnos;

- que las frases sean de uso frecuente, o más conocidas, por los alumnos;

- de esas 73 frases las profesoras deberían elegir 60 frases que considerasen bien elaboradas y más frecuentes.

4) Después de ese análisis, fueron seleccionadas las 60 frases consideradas bien elaboradas, las cuales después fueron sometidas a una prueba preliminar de comprensión de oraciones, aplicadas a 12 alumnos de enseñanza fundamental de $4^{\mathrm{a}}$, $5^{\mathrm{a}}, 6^{\mathrm{a}}$ y $7^{\mathrm{a}}$ series (primaria). Como resultado de esa aplicación, fueron perfeccionadas algunas oraciones, de acuerdo con las sugerencias proporcionadas por los propios alumnos y por las profesoras de sala de clases consultadas.

5) Elaboración de una prueba de Comprensión de Oraciones. Previamente, perfeccionando algunas oraciones y con la finalidad de elaborar las 60 frases de manera adecuada y comprensible para escolares de $5^{\mathrm{a}}$ y $8^{\mathrm{a}}$ serie de enseñanza fundamental, consideramos pertinente construir una prueba de Comprensión de Oraciones, basada en las 60 frases ya seleccionadas y elaboradas previamente (que formarían parte del test de reading span), se agregaron otras 120 frases que funcionaron como "frases distractoras", con la finalidad de neutralizar la tendencia de los sujetos para establecer "reglas" de respuestas a las preguntas de comprensión. En ese sentido, construimos una prueba de Comprensión de Oraciones con 180 frases.

Con el propósito de saber si las frases fueron comprendidas por los sujetos, elaboramos para cada frase una pregunta de comprensión, que indujeron a responder SÍ (SIM) o NO (NÃO). Por ejemplo:

Oración: O cheiro gostoso de frango assado saía da cozinha.

Pregunta: O cheiro de frango assado vinha da cozinha?

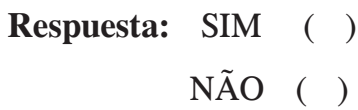

Oración: Numa casa antiga, os meninos caçaram um morcego.

Pregunta: Os meninos pescaram um morcego? 


\section{Respuesta: SIM （ )}

NÃO ( )

De las 180 frases de la prueba, la mitad incluyó preguntas que indujeron respuestas afirmativas y la otra mitad indujeron respuestas negativas. El orden secuencial de las frases en la prueba fue establecida aleatoriamente.

6) Los resultados de la aplicación de esa prueba permitieron realizar un análisis interno de los ítems, relativo solo a las 60 frases que serían parte del test final de reading span, perfeccionando o cambiando algunas frases con otra de mejor comprensión.

Todo ese proceso de elaboración de las frases fue considerado como criterio básico para establecer el carácter comprensivo de esas oraciones. Después surgió el problema de la validez de nuestro nuevo test de reading span, esto es, si ese test mediría lo que pretende medir, o sea, capacidad de la memoria operacional. Eso constituyó otro objetivo importante de nuestro trabajo. Al respecto, cabe destacar que la elaboración del mencionado test de reading span, según el constructo teórico de la memoria operacional, viene a ser una forma de validación conocida como "validez de constructo". De modo que nuestro nuevo test de reading span cumple mínimamente con ese criterio de validación, pero no es suficiente. Entonces, consideramos necesario analizar la validez de ese nuevo test correlacionando sus puntajes con los de otra prueba que evalúe procesos cognitivos similares al modelo de la memoria operacional, que constituiría el procedimiento de la "validez de criterio".

Como ya fue mencionado, consideramos el subtest de Memoria de Dígitos, en la forma invertida, del WISC, como el test criterio para correlacionarlo con el test reading span, elaborado en este estudio, tomando en cuenta que ambos test implicarían el componente de procesamiento de la memoria operacional, que se supone es el aspecto cognitivo principal que establece las diferencias individuales en la habilidad de la lectura.

Basados en esas referencias teóricas, sobre el modelo de la memoria operacional, que constituye el fundamento del test de reading span, en el presente estudio nos propusimos alcanzar los siguientes objetivos: a) elaborar un nuevo test de reading span según los criterios proporcionados por Daneman y Carpenter (1980), adaptado para niños escolarizados de enseñanza fundamental, b) conferir validez para el nuevo test de reading span mediante la correlación con el subtest de Memoria de Dígitos en la forma invertida del WISC, en la medida que el subtest del WISC implica el componente de procesamiento de la memoria operacional, y en consecuencia verificar la eficacia del nuevo test de reading span para establecer de manera adecuada diferencias individuales en la capacidad de lectura en niños escolarizados del nivel primario, y c) analizar las posibles influencias de las variables categóricas de escolaridad, género y tipo de escuela, en desempeño de las tareas de reading span y digit span (directa e inversa).

\section{Experimento}

Sujetos. Los sujetos fueron 163 escolares de 5a y 8a serie de enseñanza fundamental, hombres y mujeres, que asisten a escuelas estatales y particulares de la ciudad de Campinas-SP, Brasil.

Materiales. El test de reading span elaborado de acuerdo con el constructo de la memoria operacional y los criterios sugeridos en el Experimento 1 de Daneman y Carpenter (1980), 
adaptado para escolares de enseñanza fundamental. Los ítems son 60 oraciones sin relación una con la otra, compuestas de 7 a 10 palabras. Las frases terminan con substantivos de tres sílabas, que fueron seleccionadas de textos que tienen una razonable frecuencia de consulta y uso en las tareas escolares, y el carácter comprensivo de esas frases fue conferido por medio de una prueba de Comprensión de Oraciones.

Ejemplos de dichas frases (en portugués):

Perto de uma piscina, umas garotas tomavam sorvete.

No parque havia um trem que soltava muita fumaça.

Cada una de las frases fue colocada en una única línea, al centro de una tarjeta de $15 \mathrm{x}$ $20 \mathrm{~cm}$. Las oraciones fueron organizadas en cinco grandes grupos: el primer grupo con tres subgrupos con dos oraciones cada uno, el segundo grupo con tres subgrupos con tres oraciones cada uno, el tercer grupo con tres subgrupos con cuatro oraciones cada uno, el cuarto grupo con tres subgrupos con cinco oraciones cada uno, y el quinto grupo con tres subgrupos con seis oraciones cada uno. Para marcar el inicio y el fin de cada grupo fueron introducidas tarjetas en blanco. Cada grupo de frases conformó un Nivel de reading span, de modo que los niveles del test fueron: Nivel 2, Nivel 3, Nivel 4, Nivel 5 y Nivel 6.

El subtest de Números del Test Wechsler de Inteligência para Crianças (Wechsler Intelligence Scale for Children-WISC) es una prueba de retención de dígitos que presenta dos modalidades: a) Orden Directa: los números se repiten exactamente como fueron presentados. La capacidad de la memoria directa de dígitos fue definida como la mayor cantidad de dígitos repetidos correctamente por los participantes, en el orden en que fue escuchada, en uno de los dos ensayos; y b) Orden Inversa: los números se repiten de atrás para adelante. La capacidad de la memoria invertida de dígitos fue definida como la mayor cantidad de dígitos repetidos correctamente por los participantes, en el orden invertido, en por lo menos en uno de los dos ensayos.

\section{Procedimiento}

Las pruebas fueron aplicadas individualmente, en el mismo local escolar. La tarea del sujeto en relación al test de reading span fue leer en voz alta cada oración, en ritmo normal, y al final de cada grupo debía retener y evocar las palabras finales de cada oración, en el orden en que fueron expuestas. Las respuestas de los sujetos fueron anotadas en hojas de respuestas previamente preparadas. El nivel de reading span de cada sujeto fue determinado como el número del mayor grupo de oraciones cuyas palabras finales habían sido correctamente evocadas. Antes de comenzar la prueba, todos los sujetos pasaron por una etapa de entrenamiento, con la finalidad de constatar si tuvo comprensión de la tarea del mencionado test. La prueba terminó cuando el sujeto no recordó las últimas palabras de dos subgrupos de cualquier grupo de oraciones.

El subtest de Números del WISC, de la forma directa e invertida, fue administrado y evaluado de acuerdo con las indicaciones del Manual del WISC, estableciendo las puntuaciones de la memoria inmediata de dígitos de cada sujeto así como la cantidad de números de la mayor serie repetida correctamente.

El orden de aplicación de las dos baterías, test de reading span y subtest de Números del 
WISC, fue variada aleatoriamente para compensar efectos intervinientes.

\section{RESULTADOS}

En primer lugar se exponen las tablas de frecuencias y porcentajes, que muestran los niveles de reading span y de la memoria de dígitos, en la forma directa e inversa, de los sujetos de $5^{\mathrm{a}}$ y $8^{\mathrm{a}}$ series (nivel primario), distribuidos en un total de 163 sujetos. Así, en la Tabla N. ${ }^{\circ} 1$ se puede notar que la mayoría de los sujetos alcanzó el Nivel 3 de reading span, equivalente a 52,1\% del total, mientras que el Nivel 2 fue alcanzado por el 23,3\% de los sujetos, y el Nivel 4 de reading span fue obtenido por el $19 \%$.

Tabla N. ${ }^{0}$ 1. Frecuencias y porcentajes de los niveles de reading span de los sujetos de $5^{\mathrm{a}}$ y $8^{\mathrm{a}}$ series.

\begin{tabular}{ccc}
\hline $\begin{array}{c}\text { Nivel } \\
\text { Reading Span }\end{array}$ & Frecuencia & $\begin{array}{c}\text { Porcentaje } \\
(\boldsymbol{\%})\end{array}$ \\
\hline 2 & 38 & 23,3 \\
3 & 85 & 52,1 \\
4 & 31 & 19,0 \\
5 & 8 & 4,9 \\
6 & 1 & 0,6 \\
\hline Total & 163 & 99,9 \\
\hline
\end{tabular}

En relación a la capacidad de la memoria de dígitos (digit span) en la forma directa, se puede percibir en la Tabla N. ${ }^{\circ} 2$ que el $36,2 \%$ de los sujetos alcanzó a retener y evocar cinco dígitos, mientras que el 30,1\% de los sujetos logró recordar seis dígitos, y el 19,6\%, siete dígitos.

Tabla $\mathbf{N}{ }^{\circ}$ 2. Frecuencias y porcentajes de la memoria directa de dígitos de los sujetos de $5^{\text {a }}$ y $8^{\mathrm{a}}$ series.

\begin{tabular}{ccc}
\hline $\begin{array}{c}\text { Memoria Directa } \\
\text { de dígitos }\end{array}$ & Frecuencia & Porcentaje (\%) \\
\hline 4 & 21 & 12,9 \\
5 & 59 & 36,2 \\
6 & 49 & 30,1 \\
7 & 32 & 19,6 \\
8 & 2 & 1,2 \\
\hline Total & 163 & 100,0 \\
\hline
\end{tabular}

Con respecto a la capacidad de la memoria de dígitos (digit span) en la forma inversa, 
la Tabla N. ${ }^{\circ} 3$ muestra que el $42,9 \%$ de los sujetos logró recordar cuatro dígitos y el $30,7 \%$ tuvo la capacidad de recordar tres dígitos, mientras que el 18,4\% recordó cinco dígitos.

Tabla N. ${ }^{\circ}$ 3. Frecuencias y porcentajes de la memoria invertida de dígitos de los sujetos de $5^{\mathrm{a}}$ y $8^{\mathrm{a}}$ series.

\begin{tabular}{crr}
\hline $\begin{array}{c}\text { Memoria Invertida } \\
\text { de dígitos }\end{array}$ & Frecuencia & Porcentaje (\%) \\
\hline 2 & 8 & 4,9 \\
3 & 50 & 30,7 \\
4 & 70 & 42,9 \\
5 & 30 & 18,4 \\
6 & 1 & 0,6 \\
7 & 4 & 2,5 \\
\hline Total & 163 & 100,0 \\
\hline
\end{tabular}

En el análisis de la correlación de los puntajes obtenidos por los test de reading span y el subtest de memoria de dígitos, formas directa e invertida do WISC, optamos por el estadístico no paramétrico del coeficiente de correlación RHO de Spearman, dado que los puntajes del test de reading span fueron establecidos de acuerdo con los niveles ordenados de la siguiente manera: Nivel 2, Nivel 3, Nivel 4, Nivel 5 y Nivel 6. Siendo así, en la Tabla N. ${ }^{\circ} 4$ se muestra que el coeficiente de correlación entre el nuevo test de reading span y los subtest de memoria de dígitos, formas directa e inversa, son: $\mathrm{rRHO}=0,40$ y $\mathrm{rRHO}=$ 0,47 , respectivamente. Esas correlaciones son positivas y significativas (correlaciones significativas y diferentes de cero). En otros términos, se puede decir que cuando aumentan los niveles de reading span, también aumentan los puntajes de la memoria directa y de la memoria invertida de dígitos, en $40 \%$ e $47 \%$, respectivamente. Se nota que la correlación entre el reading span y la memoria invertida de dígitos es mayor que la correlación entre el reading span y la memoria directa de dígitos, lo que indicaría que tanto el nuevo test de reading span elaborado en esta investigación cuanto el subtest de memoria dígitos en la forma invertida del WISC implicarían mecanismos computacionales o de procesamientos de la información simbólica.

Tabla N. ${ }^{\circ}$ 4. Correlación de Spearman entre el test de reading span y el subtest de memoria de dígitos, formas directa e inversa.

\begin{tabular}{lccc}
\hline \multicolumn{1}{c}{ Tests } & Reading Span & Digit Span Directa & Digit Span Invertida \\
\hline Reading Span & 1,000 & $0,406^{*}$ & $0,471^{*}$ \\
Digit Span Directa & $0,406^{*}$ & 1,000 & $0,385^{*}$ \\
Digit Span Invertida & $0,471^{*}$ & $0,385^{*}$ & 1,000 \\
\hline
\end{tabular}

${ }^{*}$ Correlación significativa y diferentes de cero. 
En lo que respecta a la intervención de las variables categóricas de escolaridad (5a y 8 a serie), género (masculino y femenino) y tipo de escuela (estatal y particular), en las capacidades de lectura (reading span) y de la memoria de dígitos (digit span) en las formas directa e invertida, fue realizada en función del análisis estadístico de comparación de las medias aritméticas de los puntajes obtenidos en el test de reading span y el subtest del WISC de memoria de dígitos, directa e invertida, de acuerdo con las variables categóricas mencionadas. La comparación de los puntajes de los grupos fue hecha mediante el test no paramétrico de Mann-Whitney (Test Z). Así, en la Tabla N. ${ }^{\circ} 5$ se muestra que hay evidencia estadística para decir que existen diferencias entre los grados de instrucción en relación a los test de reading span $(\mathrm{p}=0,024)$, memoria de dígitos en la forma directa $(\mathrm{p}=0,025)$ y memoria de dígitos en la forma invertida $(\mathrm{p}=0,006)$. En consecuencia, se puede decir que el nivel de escolaridad influye en la capacidad de reading span, en la memoria de dígitos en las formas directa e invertida, favoreciendo a los sujetos de la 8 a serie.

Tabla $\mathbf{N} .^{0}$ 5. Test $\mathrm{Z}$ de comparación de los puntajes de los test reading span y memoria de dígitos directa e inversa, según la variable categórica Nivel de escolaridad (5a / 8a serie).

\begin{tabular}{lccccccc}
\hline \multirow{2}{*}{$\begin{array}{c}\text { Variables } \\
\text { categóricas }\end{array}$} & \multicolumn{2}{c}{ Test reading span } & \multicolumn{2}{c}{$\begin{array}{c}\text { Memoria Dígito } \\
\text { Directa }\end{array}$} & \multicolumn{3}{c}{$\begin{array}{c}\text { Memoria Dígito } \\
\text { Invertida }\end{array}$} \\
\cline { 2 - 8 } & \multicolumn{2}{c}{ Media } & $\mathrm{Z}$ & Media & $\mathrm{Z}$ & Media & $\mathrm{Z}$ \\
\hline \multirow{2}{*}{ Serie } & $5 \mathrm{a}$ & 2,94 & & 5,43 & & 3,65 & \\
& $8 \mathrm{a}$ & 3,21 & $-2,257^{*}$ & 5,78 & $-2,239^{* *}$ & 4,09 & $-2,757^{* * *}$ \\
\hline
\end{tabular}

* Nivel de significación: $p=0,024$

** Nível de significación: $p=0,025$

*** Nivel de significación: $p=0,006$

En la Tabla N. ${ }^{\circ} 6$ se percibe que no hay diferencias estadísticamente significativas entre niños y niñas para los test reading span, memoria de dígitos en las formas directa e invertida. Lo cual indicaría que el género no influye significativamente en las capacidades de reading span y en las capacidades de la memoria de dígitos, directa e inversa. Mientras que en la Tabla N. ${ }^{\circ} 7$ se nota que hay diferencias significativas entre las escuelas estatales y particulares en relación a los tests de reading span, memoria de dígitos directa e inversa, favoreciendo a los sujetos que frecuentan escuelas particulares, lo que estaría indicando que el hecho de asistir a escuelas particulares influye en el desempeño de las tareas de reading span y digit span (directa e invertida). 
Tabla N. ${ }^{0}$ 6. Test $\mathrm{Z}$ de comparación de los puntajes de los tests reading span y de la capacidad de memoria dígitos directa e invertida, según la variable categórica Género (masculino/femenino).

\begin{tabular}{lccccccc}
\hline \multirow{2}{*}{$\begin{array}{c}\text { Variables } \\
\text { categóricas }\end{array}$} & \multicolumn{2}{c}{ Test Reading Span } & \multicolumn{2}{c}{$\begin{array}{c}\text { Memoria Dígito } \\
\text { Directa }\end{array}$} & \multicolumn{3}{c}{$\begin{array}{c}\text { Memoria Dígito } \\
\text { Invertida }\end{array}$} \\
\cline { 2 - 8 } & Media & $Z$ & Media & $Z$ & Media & $Z$ \\
\hline \multirow{2}{*}{ Género } & $\mathrm{M}$ & 3,17 & & 5,71 & & 3,98 & \\
& $\mathrm{~F}$ & 3,97 & $-1,739$ & 5,48 & $-1,534$ & 3,75 & $-1,496$ \\
\hline
\end{tabular}

Tabla N. ${ }^{0}$ 7. Test $\mathrm{Z}$ de comparación de los puntajes de los tests reading span y de la capacidad de memoria de dígitos directa e invertida, según la variable categórica Tipo de escuela (estatal/ particular).

\begin{tabular}{|c|c|c|c|c|c|c|c|}
\hline \multicolumn{2}{|c|}{$\begin{array}{l}\text { Variables } \\
\text { categóricas }\end{array}$} & \multicolumn{2}{|c|}{ Test Reading Span } & \multicolumn{2}{|c|}{$\begin{array}{c}\text { Memoria Dígito } \\
\text { directa }\end{array}$} & \multicolumn{2}{|c|}{$\begin{array}{c}\text { Memoria Dígito } \\
\text { invertida }\end{array}$} \\
\hline & & Media & $Z$ & Media & $Z$ & Media & $Z$ \\
\hline \multirow[t]{2}{*}{$\begin{array}{l}\text { Tipo de } \\
\text { Escuela }\end{array}$} & E & 2,84 & & 5,35 & & 3,65 & \\
\hline & $\mathrm{P}$ & 3,30 & $-3,800 *$ & 5,85 & $-3,203^{*}$ & 4,07 & $-2,885^{*}$ \\
\hline
\end{tabular}

* Nivel de significación: $p<0,05$

\section{DISCUSIÓN}

Teniendo en cuenta el propósito de establecer la validez de nuestro nuevo test de reading span, los puntajes resultantes de su aplicación fueron correlacionados con los puntajes del subtest de memoria de dígitos, forma directa e inversa del WISC. También fue examinada la posibilidad de que las variables categóricas de Escolaridad, Género y Tipo de escuela, influenciaran en los desempeños de reading span y digit span (directa e invertida).

En el análisis general, el desempeño en la tarea de reading span del grupo total de participantes $(n=163)$ varió entre el Nivel 2 y el Nivel 6 de reading span, obteniendo en media 3,07 de nivel de reading span, lo cual fue distinto de los resultados obtenidos por la aplicación original del test de reading span, según Daneman y Carpenter (1980), donde el nivel de reading span varió entre 2 y 5, con una media de 3,18 (en un grupo de 20 estudiantes universitarios de pregrado). Al respecto, Just y Carpenter (1992) sugirieron una clasificación general de la capacidad en la lectura (reading span), basada en los puntajes obtenidos en el test original de reading span de Daneman-Carpenter, como sigue:

- $\quad$ Alto reading span: de 4 a más palabras (hasta 5,5 palabras)

- $\quad$ Medio reading span: de 3 a 3,5 palabras

- Bajo reading span: menos de 3 palabras

Referente a la correlación entre nuestro nuevo test de reading span y el subtest de memoria de dígitos (digit span), en la forma directa e inversa del WISC, se utilizó el coeficiente 
de correlación de Spearman, comprobándose correlaciones positivas estadísticamente significativas: $\mathrm{rRHO}=0,40$ y $\mathrm{rRHO}=0,47$, respectivamente. La correlación que nos interesó en este análisis fue la segunda, entre el test de reading span y el digit span inverso $(\mathrm{rRHO}=0,47)$. Esa correlación positiva significativa estaría indicando que ambos test implicarían mecanismos computacionales y de procesamiento de la información simbólica, que es un componente importante de la memoria operacional (Jensen \& Figueroa, 1975; Letho, 1996; Schofield \&Ashman, 1986; Sü $\beta$, Oberauer, Wittmann, Wilhelm \& Schulze, 2002; Waters \& Caplan, 1996). En ese sentido, podemos afirmar que el nuevo test de reading span, que hemos elaborado y aplicado en este trabajo de investigación, tiene validez.

En base a esos resultados, podemos inferir que nuestro nuevo test de reading span, además de activar la función cognitiva de procesamiento, también fomenta la activación simultánea de la memoria a corto plazo (entendido como un buffer de almacenamiento), funciones que están implicados en esos test (reading span y digit span, directa e invertida), lo que constituiría una prueba más de que la simple memoria a corto plazo es un componente que no puede faltar en la memoria operacional. Según Baddeley (1986), la memoria a corto plazo sería una función del bucle fonológico o articulatorio. Coincidiendo con esa idea, Engle et al. (1999) relataron que Cowan consideró la memoria a corto plazo como un subcomponente de la memoria operacional, además del componente de la atención controlada (Engle et al., 1999: 105; Kail y Hall, 2001).

También esos resultados están en concordancia con el presupuesto que considera a la memoria simple de corto plazo y la memoria operacional como dos constructos diferentes. En esa perspectiva, Lehto (1996) afirmó que las tareas de la capacidad de la memoria operacional pueden ser simples y complejas. La capacidad simple de la memoria operacional se refiere a tareas de digit span y word span, que se supone evalúa principalmente las funciones de almacenamiento de la memoria operacional. Mientras que la tarea compleja de la memoria operacional (e.g., test de reading span) se presume que evalúa ambas funciones de la memoria operacional: el almacenamiento y el procesamiento (Lehto, 1996: 31).

En relación a la intervención de la variable escolaridad (ver Tabla N. ${ }^{\circ}$ 5), se constató que la mayor experiencia educacional de los sujetos de $8^{\mathrm{a}}$ serie, en relación con los sujetos con menor experiencia educacional de $5^{\mathrm{a}}$ serie, benefició la obtención de mayores puntajes en las tareas de reading span y digit span, directa e invertida. Esa evidencia sugeriría que la variable cultural (expresada por el nivel de escolaridad) estaría en relación con la posesión de mayor información y conocimiento de los alumnos de $8^{\mathrm{a}}$ serie, que habrían afectado favorablemente en los resultados de las tareas de reading span y digit span, en la forma directa e invertida, en la medida que esas tareas implicarían habilidades en la lectura y en la capacidad de memorización, adquiridas por la experiencia escolar.

Con respecto al género (ver Tabla N. ${ }^{\circ}$ 6), se observó que en los puntajes de los niños y niñas no existe diferencia significativa, en relación a las tareas de reading span y digit span en la forma directa e invertida, de modo que eso indicaría que la diferencia de género es un factor que no influiría en el desempeño de las tareas mencionadas.

Referente al tipo de escuela frecuentada por los sujetos (ver Tabla N. ${ }^{\circ}$ 7) se observó que los que asisten a escuelas particulares superan significativamente, en todas las tareas 
administradas en este estudio (reading span y digit span, forma directa e inversa), a los sujetos que asisten a escuelas estatales. Eso indica que el estatus socioeconómico de los sujetos estudiados es un factor significativo que estaría influyendo en las habilidades de lectura y memoria de dígitos (directa e invertida).

\section{CONCLUSIONES}

De acuerdo con uno de los propósitos principales de este estudio, se elaboró una nueva versión del test de reading span, adaptado para niños escolarizados de enseñanza fundamental, según los criterios sugeridos por Daneman y Carpenter (1980). Ese nuevo test está compuesto por frases con términos del vocabulario que son de uso frecuente en los niños escolarizados del nivel educativo de enseñanza fundamental de la ciudad de Campinas, del Estado de San Pablo, Brasil. Cabe mencionar que esas frases fueron seleccionadas después de una prueba rigurosa de comprensión de oraciones, realizada en un grupo representativo de niños escolarizados de enseñanza fundamental de la mencionada ciudad brasilera.

Respecto a la validación del nuevo test de reading span, se procedió a correlacionarlo con el subtest de Memoria de Dígitos en la forma invertida del WISC (al que también se le denominó digit span invertida), dando como resultado una correlación positiva significativa, esto es rRHO $=0,47$; lo cual reforzó nuestra hipótesis de que ambos test implicarían el componente procesamiento de la memoria operacional, lo que proporcionaría validez para el nuevo test de reading span que hemos elaborado. Siendo así, ese test estaría habilitado para establecer diferencias individuales en la capacidad de lectura en sujetos que tengan características similares a los del grupo estudiado.

En relación a las variables categóricas de escolaridad, género y tipo de escuela, como posibles factores intervinientes en el desempeño de las tareas de reading span y digit span (directa e invertida), se constató que la experiencia educacional (nivel de escolaridad) es una variable influyente en el desempeño de las tareas de reading span y digit span directa e invertida. Esa evidencia coincidiría con la hipótesis de Cole (1999) quien señaló que la experiencia cultural constituye un factor que afecta los procesos psicológicos superiores. En este caso la capacidad en la lectura (reading span) es un proceso cognitivo superior que estaría influenciada por la experiencia cultural. En ese sentido, los niños estudiados de $8^{\mathrm{a}}$ serie de enseñanza fundamental demostraron mayor desempeño en la tarea de reading span, que los niños de $5^{\text {a }}$ serie, obviamente debido a la mayor experiencia educacional, o cultural, de los escolares de $8^{\mathrm{a}}$ serie, en relación a la menor experiencia educacional, o cultural, de los escolares de $5^{\text {a }}$ serie.

Referente a la variable género se constató que es un factor neutral para influenciar en las tareas de reading span y digit span, directa e invertida, lo que indicaría que las habilidades en esas tareas no dependerían del hecho de ser niño o niña, ya que ambos géneros demostraron habilidades semejantes en las tareas mencionadas. Respecto de la variable tipo de escuela se constató que los niños de las escuelas particulares superaron en las habilidades de reading span y digit span directa e invertida, a los niños de las escuelas estatales. Por tanto, se comprobó que el estatus socioeconómico es una condición 
influyente en las habilidades cognitivas superiores de lectura y memoria de dígitos en la forma invertida, y en las habilidades simples de memoria de dígitos en la forma directa.

\section{REFERENCIAS BIBLIOGRÁFICAS}

1. Atkinson, R. C. y Shiffrin, R. M. $(1968,1983)$. Memoria humana: Una propuesta sobre el sistema y sus procesos de control. En Ma. Victoria Sebastián (comp.) Lecturas de psicología de la memoria. Madrid: Alianza Editorial.

2. Baddeley, A. (1986). Working memory. New York: Oxford University Press.

3. Baddeley, A. (2000). The episodic buffer: a new component of working memory? Trends Cognition Science, pp. 417-423.

4. Baddeley, A. y Hitch, G. (1983). Memoria en funcionamiento [1974]. En: María Victoria Sebastian (Comp.), Lecturas de psicología de la memoria. Madrid: Alianza Editorial.

5. Baddeley, A., Logie, R., Nimmo-Smith, I. and Brereton, N. (1985). Components of fluent reading. Journal of Memory and Language, 24: 119-131.

6. Cain, K., Oakhill, J., and Bryant, P. (2004). Children's Reading Comprehension Ability: Concurrent Prediction by Working Memory, Verbal Ability, and Component Skills. Journal of Educational Psychology 96 (1): 31-42.

7. Caplan, D., and Waters, G. S. (1999). Verbal working memory and sentence comprehension. Behavioral and Brain Science, 22: 77-126.

8. Cole, M. (1999). Culture-Free versus Culture-Based Measures of Cognition. En Robert J. Sternberg (Ed.) The nature of cognition. MA.: The MIT Press.

9. Conway, A. R. A., \& Engle, R. W. (1996). Individual differences in working memory.

10. Capacity: More Evidence for a General Capacity Theory. Memory, 4 (6): $577-$ 590.

11. Cowan, N. (1999). An embedded-processes model of working memory. En: Akira Miyake \& Priti Shah (Eds.) Models of working memory: Mechanisms of active maintenance and executive control. Cambridge: Cambridge University Press.

12. Daneman, M. and Carpenter, P. A. (1980). Individual differences in working memory and reading. Journal of Verbal Learning and Verbal Behavior, 19: 450-466.

13. Daneman, M. and Merikle, P. M. (1996). Working memory and language comprehension: A Meta-analysis. Psychonomic Bulletin \& Review, 3 (4): 422-433.

14. Dixon, P., LeFevre, J-A. and Twilley, L. C. (1988). Word knowledge and working memory as predictors of reading skill. Journal of Educational Psychology, 80 (4): 465-472. 
15. Engel, P. M. J., Santos, F. H., Martin, R. and Gathercole, S. E. (2007). Are working memory measure free of socio-economic and cultural influences? Abstracts da International Meeting: Working memory and its relations with other memories. São Paulo: Universidade Federal de São Paulo.

16. Engle, R. W., Kane, M. J., and Tuholski, S. W. (1999). Individual differences in working memory capacity and what they tell us about controlled atenttion, general fluid intelligence, and functions of the prefrontal-cortex. En: Akira Miyake and Priti Shah (Eds.) Models of working memory: mechanisms of active maintenance and executive control. Cambridge: Cambridge University Press.

17. Engle, R. W. and Oransky, N. (1999). Multi-Store versus dynamic models of temporary storage in memory. En: Robert J. Sternberg (Ed.) The nature of cognition. MA: The MIT Press.

18. Fedorenko, E., Gibson, E., and Rhode, D. (2006). The nature of working memory capacity in sentence comprehension: Evidence against domain-specific working memory resources. Journal of Memory and Language, 54: 541-553.

19. Friedman, N. P., and Miyake, A. (2004). The reading span test and its predictive power for reading comprehension ability. Journal of Memory and Language, 51: 136-158.

20. Grigorenko, E. L. (1999). Heredity versus environment as the basis of cognitive ability. En: Robert J. Sternberg (Ed.) The nature of cognition. MA.: The MIT Press.

21. Jensen, A. R., and Figueroa, R. A. (1975). Forward and backward digit span interaction with race and IQ: Predictions from Jensen's Theory. Journal of Educational Psychology, 67 (6): 882-893.

22. Just, M. A. and Carpenter, P. A. (1992). A capacity theory of comprehension: individual differences in working memory. Psychological Review, 99 (1): 122-149.

23. Just, M. A. and Carpenter, P. A., and Keller, T. A. (1996). The capacity Theory of Comprehension: New frontiers of evidence and arguments. Psychological Review, 103 (4): 773-780.

24. Kail, R. and Hall, L. K. (2001). Distinguishing short-term memory from working memory. Memory \& Cognition 29 (1): 1-9.

25. King, J. and Just, M. A. (1991). Individual differences in syntactic processing: The role of working memory. Journal of Memory and Language, 30: 580-602.

26. Lehto, J. (1996). Are executive function tests dependent on working memory capacity? The Quarterly Journal of Experimental Psychology, 49 A (1): 29-50.

27. Lima, P. L. C. y Françozo, E. (2001). Memória operacional e Produção Oral da Linguagem. Cadernos de Estudos Lingüísticos, UNICAMP, 40: 93-105.

28. MacDonald, M. C., Just, M. A., and Carpenter, P. A. (1992). Working memory constraints on the processing of syntactic ambiguity. Cognitive Psychology, 24: 56-98. 
29. Masson, M. E. J., and Miller, J. A. (1983). Working Memory and individual differences in comprehension and memory of text. Journal of Educational Psychology, 75 (2): 314-318.

30. Miyake, A., and Shah, P. (1999). Models of Working Memory: Mechanisms of Active Maintenance and Executive Control. Cambridge: Cambridge University Press.

31. Ruchkin, D. S., Grafman, J., Cameron, K., and Bernt, R. S. (2003). Working memory retention systems: A state of activated long-term memory. Behavioral and Brain Sciences, 26: 709-777.

32. Santos, F. H. (2007). Working Memory, Episodic Memory and Mathematical Skills in Children. Abstracts da International Meeting: Working Memory and Its Relations with Other Memories. São Paulo: Universidade Federal de São Paulo.

33. Schofield, N. J., and Ashman, A. F. (1986). The Relationship between Digit Span and Cognitive Processing across Ability Groups. Intelligence, 10: 59-73.

34. Süß, H-M., Oberauer, K., Wittmann, W. W., Wilhelm, O., and Schulze, R. (2002). Working-memory capacity explains reasoning ability -and a little bit more. Intelligence, 30: 261-288.

35. Turner, M. L., and Engle, R. W. (1989). Is working memory capacity task dependent? Journal of Memory and Language, 28: 127-154.

36. Waters, G. S., and Caplan, D. (1996). The measurement of verbal working memory capacity and its relation to reading comprehension. The Quarterly Journal of Experimental Psychology, 49A (1): 51-79. 